\title{
A Comparison of Stem Cell Therapies for Parkinson Disease
}

\author{
Simon Hwang ${ }^{1}$, Sunydip Gill', Seema Pathak', Samyuth Subramanian \\ ${ }^{1}$ Georgetown University School of Continuing Studies \\ Keywords: stem cells, parkinson disease \\ https://doi.org/10.52504/001c.3420
}

Georgetown Medical Review

Vol. 2, Issue 1, 2018

\begin{abstract}
Parkinson disease (PD) is a neurodegenerative disease characterized by the degeneration of dopaminergic neurons in the substantia nigra pars compacta. Current treatment options for patients with PD include levodopa and deep-brain stimulation. These treatments, although at times effective, are not a panacea for individuals with PD because they only alleviate symptoms. Recently, many different stem cell techniques have emerged that show promise at effectively curing PD, including neural stem cells, induced pluripotent stem cells, and adipose-derived mesenchymal stem cells. Although these techniques show potential, they are limited by the lack of in vivo human testing and clinical trials in animal models of PD. An analysis of these 3 novel stem cell techniques shows that induced pluripotent stem cell treatment has the most promising future as the source of a cure for PD owing to its functionality in drug screening and modeling as well as its usefulness in cell replacement therapy. While still in the preliminary stages of research, stem cells provide a potential curative solution with minimal risks and deficiencies.
\end{abstract}

\section{INTRODUCTION}

In the United States, approximately 50,000 people are diagnosed as having Parkinson disease (PD) each year, while half a million people currently live with the disease. ${ }^{1} \mathrm{PD}$ is characterized by the degeneration of dopaminergic (DA) neurons in the substantia nigra pars compacta. ${ }^{2}$ Dopamine plays a significant role in voluntary movement, and the degeneration of DA neurons causes motor symptoms such as akinesia, resting tremors, and muscle rigidity. For many years, treatment of PD has involved management of symptoms and control of dopamine levels, but these treatments have not been an effective cure for individuals with later-stage PD.

A variety of $\mathrm{PD}$ treatments are currently available, including pharmacotherapy with levodopa and deep-brain stimulation. ${ }^{3,4}$ Patient response to levodopa is often variable and found to be beneficial in some cases but minimally effective in others. Specifically, levodopa treats the stiffness, tremors, spasms, and poor muscle control caused by PD, although there is an inevitable decline with disease progression in all patients. Some adverse effects do emerge such as hypertension, seizures, insomnia, and mood changes. The causes of this variability include inadequate dosage, inadequate dopamine formation, loss of striatal dopamine receptors, loss of output pathways from the striatum, and loss of other motor pathways. ${ }^{5}$ Deep-brain stimulation has been shown to significantly improve motor function in patients with $\mathrm{PD}$ while reducing dyskinesia, curbing many of the deficits of levodopa therapy. ${ }^{5,6}$ Although not without its risks, deep brain stimulation in certain studies has resulted in 


\begin{tabular}{|c|c|c|}
\hline Stem Cell Type & Advantages & Disadvantages \\
\hline Neural & $\begin{array}{l}\text { - Ability to differentiate into multiple cell } \\
\text { types } \\
\text { - Low risk of tumorigenesis }\end{array}$ & $\begin{array}{l}\text { - Variable study results } \\
\text { - Difficult to obtain } \\
\text { - Not able to restore complete brain function on its own }\end{array}$ \\
\hline Induced pluripotent & $\begin{array}{l}\text { - } \text { Relatively easy to obtain } \\
\text { - } \text { typility to differentiate into multiple cell } \\
\text { - } \text { Low risk of immune rejection } \\
\text { - } \text { Maintained in vitro for multiple } \\
\text { - } \text { generations } \\
\text { - } \text { Disle to completely restore brain function } \\
\text { - } \text { Drug/toxicity profiling }\end{array}$ & $\begin{array}{l}\text { - Tumorigenicity } \\
\text { - Low efficiency }\end{array}$ \\
\hline Adipose derived & $\begin{array}{l}\text { - } \quad \text { Easily collected from adipose tissue } \\
\text { - } \text { Low risk of tumorigenesis } \\
\text { - } \text { Low risk of immune rejection } \\
\text { - Safe intravenous administration } \\
\text { - } \quad \text { Neuroprotective and possible } \\
\text { neurogenesis }\end{array}$ & $\begin{array}{l}\text { - Only animal models, no clinical trials } \\
\text { - Uncertain differentiation into either neuronal or } \\
\text { mesenchymal } \\
\text { - Not able to restore complete brain function on its own }\end{array}$ \\
\hline
\end{tabular}

almost $10 \%$ of the patients to experience severe complications, such as hemorrhage and persistent symptoms, while $60 \%$ of the patients show marked improvement in motor functions. ${ }^{4}$

Although the evolution of PD treatments seems to be progressing well with deep-brain techniques, there are far too many unknowns at this point to regard these techniques as a cure for PD. The specific mechanism of brain stimulation remains undiscovered and different areas of the brain have yet to be compared with the main deep-brain stimulation treatments done on the subthalamic nucleus and the globus pallidus pars interna. ${ }^{4}$ In addition, deep-brain stimulation is an invasive and inherently risky procedure, and the cost of brain surgery can deter many potential candidates from receiving treatment. PD treatment must evolve to include an effective treatment method that is both less invasive and easily accessible. Different stem cell treatments, including neural, induced pluripotent, and adipose-derived mesenchymal cells, seem to be novel PD treatment methods that circumvent the pitfalls of levodopa and deep-brain techniques.

\section{POTENTIAL STEM CELL TREATMENT OPTIONS Neural Stem Cells}

In this review of current stem cell transplantation treatments for PD, we first consider neural stem cells (NSCs). NSCs are multipotent cells capable of differentiating into both neurons and glial cells of the nervous system. Recent studies have indicated that certain NSCs persist in the adult nervous system and are capable of regenerating new neurons. Compared with pluripotent stem cells, these multipotent NSCs exhibit higher cellular survival rates and lower risk of teratoma formation. ${ }^{3}$ In addition to the fetal isolation of NSCs, these cells can be obtained from areas of the adult brain including the subventricular 
zone, the subgranular zone, and the hippocampus. ${ }^{7}$ Acquisition of NSCs from nonfetal sources avoids the ethical issues that are presented by embryonic stem cells. Because of the abilities of NSCs to self-renew and differentiate into many types of neurons, including those that are dysfunctional in neurodegenerative diseases, their potential use in the treatment of patients with PD holds promise. $^{8}$

As previously noted, one of the major problems in PD is the inability of the substantia nigra to produce dopamine. many studies on NSCs and their application in treating PD have focused on the damaged or dysfunctional DA neurons as targets for stem cell transplantation. ${ }^{3}$ To demonstrate the capability to replace the damaged DA neurons, NSCs must first show the ability to differentiate into DA neurons. Studies have been done using forebrain-derived human NSCs (FD-hNSCs) to determine the biochemical signals necessary for differentiation of hNSCs into DA neurons. In an in vitro study, scientists cultured FD-hNSCs and treated them with combinations of bone morphogenetic protein -7 , pramipexole, and various growth factors: acidic fibroblast factor, forskolin, and phorbol-12-myristate-13-acetate. 9 Measurements of the levels of typical gene products produced by DA neurons was used to determine the level of successful differentiation. When compared with the control, FD-hNSCs treated with bone morphogenetic protein-7, pramipexole, and growth factors showed significantly increased gene expression of various products associated with DA neurons including tyrosine hydroxylase, an enzyme involved in the production of dopamine. The study also found that the levels of both basal and evoked dopamine released by the treated FD-hNSCs were increased compared with the control groups. While this particular study did not evaluate the ability of the newly cultured DA neurons to be engrafted into live animals, it did demonstrate the in vitro ability for FD-hNSCs to differentiate into viable DA neurons. ${ }^{\text {? }}$

For NSCs to be an effective treatment of neurodegenerative diseases such as $\mathrm{PD}$, their survivability and therapeutic effectiveness in vivo must be demonstrated. Researchers conducting clinical trials have shifted their interest from transplantation of NSC-derived DA neurons to neuroprotection. ${ }^{3}$ While further research is necessary before clinical trials demonstrate the viability of NSC-derived DA neurons, a future wherein curative NSC transplantation replaces damaged DA neurons may not be far off. However, NSCs currently hold promise in their modulatory and supportive role of the host neurons after transplantation. Encouraging results focusing on the ability of modified NSCs to induce the host environment to produce neurotrophins (a family of proteins that support neuron development and function) are emerging. ${ }^{10}$ In a study by Ebert et al, ${ }^{11}$ NSCs were isolated and modified to produce either glial cell line-derived neurotrophic factor (GDNF) or insulin-like growth factor (IGF-1), both known to have neuroprotective qualities. In the study, the modified NSCs were transplanted into the striatum of a rat model of PD. The results revealed that IGF-1 increased the overall survival of the implanted NSCs 
while GDNF protected fibers in the striatum expressing tyrosine hydroxylase. The study demonstrated that instead of replacing damaged DA neurons, NSCs can have a trophic effect on their in vivo microenvironment and reduce some of the symptoms of PD in rat models. ${ }^{11} \mathrm{~A}$ similar study ${ }^{10}$ examined the neuroprotection transplanted NSCs provided to their host microenvironment. In the animal study, human NSCs were transplanted into the striatum of mice and subjected to an insult injection of 1-methyl-4-phenyl-1,2,3,6-tetrahydropyridine. Not only did motor skills improve in the mice that received the transplantation, but NSCs also increased the expression of host-derived growth factors, such as IGF-1 and GDNF, when compared with the control. This finding suggests that, in addition to the neuroprotection, transplanted NSCs may stimulate their host environment to accommodate them. ${ }^{10}$ These recent findings support the use of NSCs as modifiers of their host environment rather than as replacements for degenerate DA neurons in PD.

An evaluation of the safety of NSC transplantation is also important in determining the cells' potential use in the treatment of PD. A study using animal models indicated that NSCs induced from embryonic stem cells can be successfully engrafted into the striatum to alleviate symptoms of PD. ${ }^{12}$ While this study provided proof of principle, human clinical trials remain invaluable when evaluating the safety of new treatment options. A study by Lige et $\mathrm{al}^{13}$ examined the potential adverse effects of NSC transplantation into patients diagnosed as having PD. The study used NSCs derived from embryonic stem cells to transplant into the patients, ranging from 42 to 79 years of age. For up to 57 months after surgery, no tumor formation or immune rejection was found. In addition, there were no graft-induced or delivery-related adverse effects. ${ }^{13}$ While this particular study seemed to indicate transplantation success, other studies have shown variable outcomes. Most studies have reported that patients experienced moderate improvement, while others have described less well tolerated transplantation procedures. ${ }^{3}$ Reasons for this variability include difficulties obtaining fetal sources and scarcity of adult sources of NSCs. ${ }^{3}$ Research into improved stem cell harvesting techniques, as well as procedures that decrease the cellular mortality rate, would produce more consistent study outcomes. While some clinical studies have shown minimal safety issues, additional studies are needed before reliable NSC transplantation can be made a clinically available treatment of PD.

\section{Induced Pluripotent Stem Cells}

Induced pluripotent stem cells (iPSCs) are adult somatic cells that are converted into pluripotent cells via the introduction of specific transcription factors that are found in normal pluripotent stem cells. The cells can then be differentiated into most somatic cell types and are self-renewable. In addition, iPSC can easily be used to create a cell line that is matched to the adult without resorting to cloning that can cause abnormal karyotypes and teratomas. Initially, the transcription factors were inserted by viruses, which made the 
resulting iPSCs unsuited for therapeutic treatments because of the increased tumorigenicity. However, recent research has circumvented the issue by using either modified mRNAs, proteins, or microRNAs. ${ }^{14}$ The safety of these therapeutic treatments in humans has not yet been tested; however, research in nonhuman primate models has shown that iPSC grafts are capable of returning function to parkinsonian-afflicted areas. ${ }^{15}$

In regard to PD, iPSCs are useful in 3 ways: disease modeling, drug screening, and cell replacement therapy. ${ }^{14}$ iPSCs are able to accurately model the gradual loss of DA neurons unlike parkin gene knockout models. Mutation of the parkin gene causes autosomal recessive juvenile parkinsonism. This model does not exhibit robust signs of parkinsonism; however, the model is still used because gene knockout models are much easier to cultivate than iPSCs. ${ }^{15}$ The iPSCs are generated from the patient's somatic cells and hence have an identical genetic makeup. ${ }^{14}$ This means that researchers can observe the onset and progression of the disease when comparing healthy patients' iPSCs with the iPSCs of patients with PD. Also, iPSCs from patients with PD exhibit defects not observed in those from healthy patients, giving novel insights into the specific pathophysiology. ${ }^{16}$ For example, recent research with iPSCs has linked epigenetic deregulation with monogenic and sporadic PD. ${ }^{17}$ In addition, iPSCs can be differentiated into not only DA neurons, but also astrocytes, which are also etiologically related to PD. ${ }^{14}$ This study implies that cell replacement therapy can potentially be used to replace DA neurons and the surrounding glial cells as a support to treat patients with PD.

While animal models are currently used to test drugs and toxicity, iPSCs offer a better alternative. Animals and humans have significant differences in nervous system development that make translating drug efficacy to human disease difficult and expensive. ${ }^{14}$ This means that iPSCs from humans offer better understanding of human nervous system biology than animal models. Additionally, toxicity screenings can be done across different cell types through the use of iPSCs. Drugs that affect DA neurons may also have adverse effects on associated astrocyte cells and the differences in response to drugs can be observed through the use of iPSCs. iPSCs can also be genetically engineered to investigate how slight changes in genome affect the physiological and disease processes. ${ }^{18}$ iPSCs can be maintained in vitro indefinitely without any genetic modification and the iPSCs created "maintain morphology and pluripotency" for 20 generations. This is an improvement compared with an immortalized cell line, which involves heavy genetic modification and neuronal stem cells that cannot be maintained in vitro. Most importantly, iPSCs can be differentiated into cells that are "genetically and functionally analogous to those of interest."14

As recently as 5 years ago, direct therapeutic treatment for PD through transplantation of iPSCs was not feasible. Transplantation faced many problems such as low efficiency, use of viruses, and tendency to cause 
teratomas. ${ }^{14}$ Advancements since then have used xenogeneic materials that result in contamination by animal source pathogens and may cause humans to exhibit an immune response after transplantation. However, researchers have successfully used a xeno-free media alongside a feeder-free culture system and Cre-mediated excision of reprogramming factors to get transgene-free iPSCs at a greater efficiency than before, between $0.15 \%$ and $0.3 \% .{ }^{19}$ Although more testing is needed, especially in animal models, these data mean that iPSCs are more viable from an efficacy standpoint than current clinical treatments involving fetal NSCs. ${ }^{14}$ Recent progress in clinical treatment shows promise in animal models of PD. One study found that human iPSCs transplanted into 6-hydroxydopamine-induced PD rats improved "their functional defects of rotational asymmetry at 4, 8, 12 and 16 weeks after transplantation."20 Another study transplanted iPSC-derived DA neurons into parkinsonianinduced cynomolgus monkeys. The transplanted cells were found to survive for the 2 years the study was conducted. In addition, the transplanted iPSCs reinnervated the host brains. The transplanted DA neurons grew into the putamen and showed long-term viability. Although the results were only positive for 1 of the 3 monkeys, the study demonstrates that iPSC-derived DA neurons can be used for transplantation with long-term improvement in motor function without immunosuppression. ${ }^{15}$

\section{Adipose-Derived Stem Cells}

Therapeutic stem cell studies have often focused on the use of multipotent mesenchymal stem cells (MSCs) as opposed to embryonic stem cells, the use of which poses ethical concerns. The use of embryonic stem cells is controversial specifically because it involves the destruction of human embryos, which some people view as having the same moral status as an adult. Once it was discovered that clinically using embryonic MSCs extracted from bone marrow stroma posed problems such as "pain, morbidity, and low cell number upon harvest," researchers began to explore the possibility of using adipose tissue stroma as an alternate source for stem cells because it can be easily isolated from humans using suction-assisted lipectomy. ${ }^{21}$ In a 2002 study by Zuk et $\mathrm{al}^{22}$ the abundant processed lipoaspirate (PLA) cells were analyzed for potential stem cell applications because of their differentiation capacities in vitro. Using the characteristics and differentiation potential of bone marrow MSCs as a comparative example of successful stem cell populations, PLA cells were assessed using flow cytometry, immunofluorescence with cell surface CD marker antibodies, immunohistochemistry, spectrophotometry, polymerase chain reactions, and western blotting. It was found that the PLA cells were phenotypically similar to MSCs, with both sharing multiple CD markers on their cell surfaces; simultaneously, the PLA cells have their own functionality as they differ from bone marrow MSCs in their applications to nonhematopoietic tissue indicated by their lack of CD106 markers. Additionally, the results from spectrophotometry, polymerase chain reactions, and western blotting indicated similarity with MSCs as they both have multiple mesodermal lineage 
capacity. Clones of PLA cells, which were generally termed as adipose-derived stem cells (ADSCs) in this study and tested via immunohistochemistry, spectrophotometry, and polymerase chain reactions, also expressed osteogenic, adipogenic, and chondrogenic genes, which "strongly suggest [s] that ADSCs possess multi-potentiality and may be considered stem cells." 22 Through this experiment, it was established that pluripotent stem cells can be collected from adipose tissue and may be tested in vivo for clinical applications with consideration to their plasticity during differentiation. ${ }^{22}$

After the discovery of ADSCs, scientists sharpened their focus on the structure and function of human ADSCs (hADSCs) to better understand the prospect of using them in clinical research. One study by Tomita et $\mathrm{al}^{23}$ sought to clarify hADSC characteristics and their capability in producing neurotrophic factors in vitro as differentiated Schwann cell-like cells. Similar to the study by Zuk et $\mathrm{al}^{22}$ an analysis of the full structural profile was conducted of hADSCs collected from the abdomen, buttock, and thigh using procedures of flow cytometry, immunohistochemistry, and western blotting. As expected, the hADSCs also showed cell surface markers and multilineage differentiation capabilities similar to those on MSCs. Additionally, hADSCs were differentiated into Schwann cell-like cells and assessed for glial cell function by measuring GDNF, brain-derived neurotrophic factor levels (BDNF), and nerve growth levels with enzyme-linked immunosorbent assay and then comparing these levels with those produced by undifferentiated ADSCs and primary human Schwann cells. Interestingly, the neurotrophic levels in the differentiated hADSCs were higher than in undifferentiated ADSCs and, in the case of BDNF and GDNF, higher than primary human Schwann cells, indicating that differentiated hADSCs can effectively behave as active Schwann cell-like cells in vitro. Given this suggestion, the study went one step further to examine whether transplanted differentiated hADSCs could function in vivo, and it was found that at peripheral nerves, the cells were able to "achieve full glial function" by forming myelin and "associating with regenerating axons."23

With this concept of functioning glial cell-differentiated hADSCs in vivo, researchers began investigating how these stem cells could be administered in central nerves located in the brain. While NSCs and bone marrow-derived MSCs have been shown to prevent memory impairment through the action of BDNF and by regulating the immune response, respectively, intravenous transplantation of these stem cells proved to be impossible because of risks of tumorigenesis and immune rejection. hADSCs, on the other hand, have shown high proliferation rates, low senescence ratios, and low risk of tumorigenesis and immune rejection compared with bone marrow-derived MSCs, establishing the safety of using hADSCs intravenously. A 2012 animal study by Kim et $\mathrm{al}^{24}$ compared the safety of intravenous injection of hADSCs to the already successful intracerebral stem cell injection. They then assessed neurotrophic factor levels and endogenous neurogenesis capabilities to ensure proper and optimal function of the intravenously implanted stem cells. The 
biggest contribution of this study by far was the discovery that hADSCs can migrate into the brain when intravenously administered. Using hADSClabeled fluorescence magnetic nanoparticles, the particles were tracked in vivo as they crossed the blood-brain barrier after injection and distributed to all areas of the brain except the olfactory bulb. ${ }^{24}$ With a better understanding of ADSC structure and function, as well as how the stem cells can differentiate and travel intravenously, other researchers began analyzing the safety and efficacy of ADSCs as a possible form of therapy for patients with neural disorders using animal models.

The establishment of adipose tissue as a strong source for multipotent stem cells, recognition of in-depth research into the extensive neuronal capabilities of ADSCs, and examination of therapeutic administration were significant breakthroughs in stem cell research because they affirmed that ADSCs are acceptable for clinical use. ADSCs, as part of the MSC family, also fulfill the key requirements of effective regenerative application, including being harvested in a minimally invasive way, using multiple lineages, and being transplanted safely into systems without immune rejection. In addition to meeting these requirements, they also "reduce neuronal damage and support nerve regeneration in nerve injury models." 24 This expanded the investigation of possible therapeutic options for chronic neurodegenerative disorders, such as PD.

Recently, most articles have found that a neuroprotective effect via various mechanisms leads to successful use of ADSCs. ${ }^{25-27}$ A study by Ahmed et al ${ }^{25}$ found that intravenous ADSCs decreased transforming growth factor $-\beta$ and monocyte chemoattractant protein-1 levels. Transforming growth factor- $\beta$ and monocyte chemoattractant protein-1 levels are usually low in a normal brain but are highly secreted, along with inflammatory cytokines, in injured brains. This control over the secretion of these growth factors and cytokines suggests that ADSCs display immunomodulatory behavior. Furthermore, DA neurons express various neurotrophic factors such as BDNF. In PD, the loss of DA neurons within the substantia nigra causes a decrease in BDNF as well as loss of overall striatal dopamine content. Yet, the introduction of ADSCs causes an increase in BDNF, which activates a promoter of the TH gene, upregulating the production of dopamine to make up for neuron loss. ${ }^{25} \mathrm{~A}$ study by Schwerk et $\mathrm{a}^{26}$ on rats focused on the increase of neurogenesis by hADSCs. The study found that within 3 days of transplantation of hADSCs in a subventricular zone severely affected by DA neuron loss, there was an increase in BDNF levels, thereby marking stem cell activity. Moreover, the study reported $S 100 \beta$ protein expression after implantation, which "promotes cell proliferation, migration and neuronal survival." 26 The presence of endothelial-like ADSCs was discovered close to blood vessels, which researchers linked with improved neuron and ADSC survival as well as an adaptation to a potentially harmful microenvironment. ${ }^{26}$ In 2015 , an hADSC and PD study ${ }^{27}$ on mice confirmed the neuroprotective nature of 
intravenously injected ADSCs through its assessment of mitochondrial damage and respiratory function. Using positron emission tomographic imaging, it was found that hADSCs saved $T H$-positive DA neurons from cell death by inducing a change in dopamine D2 receptor binding and stimulating increase of dopamine within the striatum. hADSC injections also decreased the number of damaged mitochondria and improved mitochondrial complex I activity to previously normal levels. ${ }^{27}$ The neuroprotective nature of ADSCs and their supposed part in neurogenesis have led researchers to believe that ADSCs would prove to be a safe treatment for PD. ${ }^{26}$

Other experiments have been focusing on treating PD with combined therapies that include ADSCs to target long-term behavioral improvement. According to Choi et al, ${ }^{27}$ intravenous hADSCs improved motor and behavioral performances. A study by Zhou et $\mathrm{al}^{28}$ performed on hemiparkinsonian rhesus monkeys analyzed the value of gene therapy using a vector encoding the $T H$ gene and a trophic factor enhancing neuron survival in conjunction with neuronal-primed ADSCs. Over 4 months, it was found that transplanting both the gene vector and trophic factor ameliorated behavioral symptoms when compared with $T H$ gene vector transplantation alone. ${ }^{28}$ Although still in the early phases of basic science research, the use of ADSCs in animal models of PD overall seems to demonstrate a promising solution to "alleviate the symptoms of the disease ...or slow the progression" in humans. 27 While other dopamine-related drugs and procedures, such as deep-brain stimulation, are effective therapies for patients with PD, recent animal studies have shown that PD improvement due to ADSCs can be equally or more successful in comparison with currently administered reference drugs. 25

\section{DISCUSSION}

$\mathrm{PD}$ is a neurodegenerative disorder that affects the quality and duration of people's lives. Any mammal that needs to produce dopamine to survive can develop PD, but numerous stem cell techniques used in smaller mammals, such as mice, rats, and nonhuman primates, have proven the potential of restoring normal DA neuron function to treat and cure PD. NSCs, iPSCs, and ADSCs have all been shown to effectively alleviate many of the deleterious symptoms in animals with PD by restoring proper dopamine generation, functioning without deleterious consequences, and decreasing levels of harmful intermediates associated with PD.

NSCs have shown promise because of their neurotrophic support of surrounding neurons to directly alleviate symptoms of PD. ${ }^{10}$ Amongst all the currently available stem cell techniques for curbing the effects of PD, NSCs seem to be the most effective at stabilizing neurotrophin levels in animal models. However, NSC studies have indicated variable transplantation success, thus, more reliable clinical applications require additional research. Problems stemming from difficulties in harvesting and high cellular mortalities have hindered transplantation procedure results. ${ }^{3}$ Although there has been 
interesting and promising progress, NSCs must be able to reliably function safely and effectively in humans if they are to become the predominant PD treatment method.

ADSCs circumnavigate many of the pitfalls of other techniques, especially by the low risk of tumorigenesis and the safety of harvesting these stem cells. ${ }^{24}$ Their ability to cross the blood-brain barrier via intravenous administration and their glial cell differentiation capabilities have demonstrated potential in the use of ADSCs in viable PD therapies. ${ }^{23,24}$ ADSCs can control many of the harmful byproducts associated with PD, such as the overproduction of cytokines and growth factors. ${ }^{25}$ ADSCs can also protect DA neurons in 3 main ways: expression of $S 100 \beta$, rescue of $T H$-positive DA neurons, and reduction of damaged mitochondria. ${ }^{26,27}$ Despite the many different types of ADSCs and hADSCs that have been used, none seem to be able to restore normative brain function or dopamine levels in animal models with PD. Experimentation with ADSCs is very much in the early stages of research, but combining use of ADSCs with other remedies, such as gene therapy, is a promising therapy for PD.

Although all the stem cell techniques reviewed in this article are possible therapeutic options, we believe that iPSCs are the best possible PD treatment because of their viability in animal models, safety, and derivation from human cells in most experiments. ${ }^{14}$ They are part of a special class of stem cells that have been proven effective in higher mammals, such as cynomolgus monkeys. ${ }^{15}$ With all the benefits that iPSCs produce, the last major step that needs to be taken is effective human trials. Once that is completed, iPSCs may become the primary treatment method for patients with PD.

\section{CONCLUSION}

In conclusion, stem cell techniques must continue to improve and be tested in living animals and humans before becoming the next major PD treatment option. Based on the comparative evidence available as summarized in the Table, it seems that iPSCs are the most promising upcoming stem cell technique not only because of therapeutic uses, but also the applications in disease modeling and drug testing. Regardless, more clinical trials must be conducted across all potential treatment types to find a truly effective solution. 


\section{REFERENCES}

1. US Department of Health and Human Services. NIH Research Portfolio Online Reporting Tool: Parkinson's disease. https://report.nih.gov/NIHfactsheets/ViewFactSheet.aspx?.csid=109. Accessed February 22, 2017.

2. Adams RD, Van Bogaert L, Vandereecken H. Striato-nigral degeneration. J Neuropathol Exp Neurol. 1964;23:584-608.

3. Pardal R, Lopez-Barneo J. Neural stem cells and transplantation studies in Parkinson's disease. Adv Exp Med Biol. 2012;741:206-216.

4. Obeso JA, Olanow CW, Rodriguez-Oroz MC, Krack P, Kumar R, Lang AE. Deep-Brain Stimulation for Parkinson's Disease Study Group. Deep-brain stimulation of the subthalamic nucleus or the pars interna of the globus pallidus in Parkinson's disease. N Engl J Med.

2001;345(13):956-963.

5. Marsden CD, Parkes JD. Success and problems of long-term levodopa therapy in Parkinson's disease. Lancet. 1977;1(8007):345-349.

6. Rosa M, Arlotti M, Marceglia S, et al. Adaptive deep brain stimulation controls levodopainduced side effects in Parkinsonian patients. Mov Disord. 2017;32(4):628-629.

7. Wang S, Okun MS, Suslov O, et al. Neurogenic potential of progenitor cells isolated from postmortem human Parkinsonian brains. Brain Res. 2012;1464:61-72.

8. Choi DH, Kim JH, Kim SM, Kang K, Han DW, Lee J. Therapeutic potential of induced neural stem cells for Parkinson's disease. Int J Mol Sci. 2017;18(1):e224.

9. Yang H, Wang J, Wang F, et al. Dopaminergic neuronal differentiation from the forebrainderived human neural stem cells induced in cultures by using a combination of BMP-7 and pramipexole with growth factors. Front Neural Circuits. 2016;10(29).

10. Zuo F, Bao X, Sun X, et al. Transplantation of human neural stem cells in a parkinsonian model exerts neuroprotection via regulation of the host microenvironment. Int J Mol Sci.

2015;16(11):26473-26492.

11. Ebert AD, Beres AJ, Barber AE, Svendsen CN. Human neural progenitor cells over-expressing IGF-1 protect dopamine neurons and restore function in a rat model of Parkinson's disease. Exp Neurol. 2008;209(1):213-223.

12. Kang $\mathrm{X}, \mathrm{Xu} \mathrm{H}$, Teng $\mathrm{S}$, et al. Dopamine release from transplanted neural stem cells in Parkinsonian rat striatum in vivo. Proc Natl Acad Sci USA. 2014;111(44):15804-15809.

13. Lige L, Zengmin T. Transplantation of neural precursor cells in the treatment of Parkinson disease: an efficacy and safety analysis. Turk Neurosurg. 2016;26(3):378-383.

14. Li W, Chen S, Li JY. Human induced pluripotent stem cells in Parkinson's disease: a novel cell source of cell therapy and disease modeling. Prog Neurobiol. 2015;134:161-177.

15. Hallett PJ, Deleidi M, Astradsson A, et al. Successful function of autologous iPSC-derived dopamine neurons following transplantation in a non-human primate model of Parkinson's disease. Cell Stem Cell. 2015;16(3):269-274.

16. Nguyen HN, Byers B, Cord B, et al. LRRK2 mutant iPSC-derived DA neurons demonstrate increased susceptibility to oxidative stress. Cell Stem Cell. 2011;8(3):267-280. 
17. Fernández-Santiago R, Carballo-Carbajal I, Castellano G, et al. Aberrant epigenome in iPSCderived dopaminergic neurons from Parkinson's disease patients. EMBO Molecular Med. 2015;7(12):1529-1546.

18. Pei Y, Peng J, Behl M, et al. Comparative neurotoxicity screening in human iPSC-derived neural stem cells, neurons and astrocytes. Brain Res. 2016;1638:57-73.

19. Lu HF, Chai C, Lim TC, et al. A defined xeno-free and feeder-free culture system for the derivation, expansion and direct differentiation of transgene-free patient-specific induced pluripotent stem cells. Biomaterials. 2014;35(9):2816-2826.

20. Han F, Wang W, Chen B, et al. Human induced pluripotent stem cell-derived neurons improve motor asymmetry in a 6-hydroxydopamine-induced rat model of Parkinson's disease. Cytotherapy. 2015;17(5):665-679.

21. Via A, Frizziero A, Oliva F. Biological properties of mesenchymal Stem Cells from different sources. Muscles, Ligaments and Tendons Journal. 2012;2(3):154-162.

22. Zuk PA, Zhu M, Ashjian P, et al. Human adipose tissue is a source of multipotent stem cells. Mol Biol Cell. 2002;13(12):4279-4295.

23. Tomita K, Madura T, Sakai Y, Yano K, Terenghi G, Hosokawa K. Glial differentiation of human adipose-derived stem cells: implications for cell-based transplantation therapy. Neuroscience. 2013;236:55-65.

24. Kim S, Chang KA, Kim J, et al. The preventive and therapeutic effects of intravenous human adipose-derived stem cells in Alzheimer's disease mice. PLoS ONE. 2012;7(9):e45757.

25. Ahmed H, Salem A, Atta H, Ghazy M, Aglan H. Do adipose tissue-derived mesenchymal stem cells ameliorate Parkinson's disease in rat model? Hum Exp Toxicol. 2014;33(12):1217-1231.

26. Schwerk A, Altschuler J, Roch M, et al. Human adipose-derived mesenchymal stromal cells increase endogenous neurogenesis in the rat subventricular zone acutely after 6-hydroxydopamine lesioning. Cytotherapy. 2015;17(2):199-214.

27. Choi HS, Kim HJ, Oh JH, et al. Therapeutic potentials of human adipose-derived stem cells on the mouse model of Parkinson's disease. Neurobiol Aging. 2015;36(10):2885-2892.

28. Zhou Y, Sun M, Li H, et al. Recovery of behavioral symptoms in hemi-parkinsonian rhesus monkeys through combined gene and stem cell therapy. Cytotherapy. 2013;15(4):467-480. 